\title{
ONOMÁVAIN
}

Revista semestral de lingüística, filología y traducción

\section{Un modelo teórico-metodológico para investigación empírica en feedback correctivo escrito en una segunda lengua}

Methodological-theoretical model for empirical research in corrective feedback in second language writing

\section{Jorge Armando Lillo Durán}

Universidad Católica de la Santísima Concepción

Chile

\section{Anita Alejandra Ferreira Cabrera}

Universidad de Concepción

Chile

\section{(c) $\underset{\mathrm{BY}}{(} \bigodot_{\mathrm{ND}}$}

Jorge Lillo Durán: Departamento de Lenguas, Facultad de Educación, Universidad Católica de la Santísima Concepción, Chile. Correo electrónico: jlillo@ucsc.cl

Anita Ferreira Cabrera: Departamento de Español, Facultad de Humanidades y Arte, Universidad de Concepción, Chile. Correo electrónico: aferreir@udec.cl 


\section{Resumen}

Mucho se ha escrito acerca del efecto del feedback correctivo escrito (FCE) en una segunda lengua en los últimos quince años. Y aunque los primeros estudios arrojaron resultados contradictorios, recientemente un cuerpo creciente de investigación ha demostrado su efectividad en la adquisición de ciertas estructuras lingüísticas. Sin embargo, aún para muchos investigadores y profesores en producción escrita en una L2, estos estudios son poco prácticos y hasta alejados de la realidad educacional. Además, recientemente, se ha generado un cuerpo de investigación que indaga cómo la comunicación mediada por computador puede impactar y crear nuevas formas de feedback correctivo en el futuro cercano. En consecuencia, en este artículo se propone un modelo de investigación para la corrección de los errores de escritura en una L2, sustentado en la metodología robusta de los estudios de ASL, en la practicabilidad de los estudios de producción escrita y en el rol de la tecnología en la entrega de feedback correctivo escrito.

Palabras clave: adquisición de segundas lenguas; comunicación mediada por computador; escritura en una segunda lengua; estructuras lingüísticas; feedback correctivo escrito.

\section{Abstract}

Much has been written about the effect of written corrective feedback (WCF) in a second language in the last fifteen years. Even though early studies show contradictory and controversial results, a growing body of research has recently demonstrated the efficacy of written corrective feedback in the acquisition of some linguistic features. However, still for many L2 researchers and teachers of writing, this approach is not very practical and even far from a real educatio- nal context. In addition, an emerging body of studies has researched how computer mediated communication can impact new forms of corrective feedback in the near future. Consequently, in this paper we introduce a research design for error correction in L2 writing, which is based on the robust methodology of the SLA studies, the relevance of the educational context of the studies of $\mathrm{L} 2$ writing and the role of technology in corrective feedback delivery.

Keywords: computer mediated communication; linguistic features; second language acquisition; second language writing; written corrective feedback. 


\section{Introducción}

La forma en que los profesores reaccionan en torno a los textos escritos de los estudiantes en una segunda lengua ha concentrado la atención de los investigadores y profesores durante las últimas dos décadas (Ellis, 2009). Un tipo de reacción corresponde a la entrega de información o feedback acerca de un número importante de situaciones encontradas en el texto escrito como son el contenido, la organización textual, el uso apropiado del vocabulario, la aplicación de las convenciones de los tipos de textos utilizados y por último el sistema lingüístico. Este último tipo de feedback, el que se concentra en los errores lingüísticos, ha recibido mayor atención por parte de los investigadores. Estas reacciones hacia las producciones escritas no nativas es lo que se conoce como feedback correctivo escrito (Van Beuningen, 2010) o corrección de la gramática (Truscott, 1996), el tipo de feedback que se preocupa de la respuesta incorrecta o agramatical (Sheen, 2011) en los textos escritos en una L2. Debido a este creciente interés de los investigadores por el FC y a la búsqueda permanente por parte de los profesores para hacer sus prácticas de feedback más efectivas es que, en los últimos veinte años, el FC se ha transformado en un punto fundamental en la escritura en una segunda lengua.

A pesar del crecimiento y evolución en la investigación, aún existe discrepancia en torno a temas como la calidad, efectividad, la pertinencia, metodología e interpretación de resultados del FC, entre otros. Esto se debe en gran parte a que la investigación se ha ubicado en el límite de dos disciplinas, ambas con sus orientaciones teóricas y metodológicas, a saber, el campo de la producción escrita en una L2 y el dominio de la adquisición de segundas lenguas (Ellis, 2010; Van Beuningen, 2010; Ferris, 2010). Por una parte, las investigaciones en ASL se preocupan de cómo el FC puede ayudar en el desarrollo de la interlengua, examinan si el FCE facilita la adqui- sición de rasgos lingüísticos particulares a largo plazo, cómo estos se adquieren y cuántos deben ser estudiados; si el feedback debe ser implícito o explicito, y cuánta explicación metalingüística es necesaria (Bitchener, 2008; Ellis, 2009; Van Beuningen, 2010; Sheen, 2011), todo lo anterior apoyado por el paradigma cognitivo de la ASL. Por otra parte, los investigadores de escritura en una L2 se preguntan si el FC escrito ayuda a los estudiantes a mejorar la efectividad general de sus textos y a desarrollarse como escritores exitosos, concentrando sus esfuerzos en explorar el rol que tiene el FC en el desarrollo de las habilidades de revisión y edición textual. Es así como la mayoría de los expertos en producción escrita valora la adquisición de rasgos lingüísticos; sin embargo, ven que un énfasis en la corrección de un grupo limitado de estructuras es demasiado restringido como foco de enseñanza en la sala de clases (Ferris, 2010; Van Beuningen, 2010). Aunque ambas posiciones han coexistido de manera paralela y hasta cierto punto antagónicas, en los últimos años han aparecido posturas que destacan las virtudes de ambos enfoques y han encontrado un punto de equilibrio entre ellas para promover su complementación.

Además de lo señalado anteriormente, existe un área de investigación que ha venido creciendo rápidamente y que se preocupa del rol que tiene la tecnología en el aprendizaje de una L2 y cómo la innovación en esta área impacta en el feedback correctivo (Ware y Warschauer, 2006; Hyland, 2010; Sheen, 2011). Los instrumentos computacionales son una herramienta poderosa para evaluar nuevas formas de feedback y de comunicación a la vez. Y aunque esta línea de investigación es incipiente, en los estudios se destaca que este tipo de feedback denominado electrónico es necesariamente un área de investigación con beneficios potenciales para los profesores y estudiantes de segundas lenguas. Para los investigadores, la pregunta central, desde esta perspectiva, es cómo la respuesta mediada 
por computador puede imitar o incluso mejorar los resultados informados por la investigación acerca de la interacción de pares en un contexto presencial. Los estudios han comparado en el diseño las prácticas conocidas en el aula con las actividades realizadas con o a través del medio electrónico (Ware y Warschauer, 2006) en líneas de investigación que van desde el feedback humano entregado por medios tecnológicos hasta el feedback automático e inteligente.

El modelo teórico de investigación en feedback correctivo escrito que se propone en este artículo se sustenta en los estudios desarrollados por los investigadores de producción escrita, quienes se preguntan si el FCE ayuda a los estudiantes a desarrollarse como mejores escritores utilizando estrategias de revisión y edición; por los investigadores de ASL, quienes se preocupan en descubrir si el FCE ayuda a los estudiantes a adquirir ciertas estructuras lingüísticas, y por los investigadores en feedback electrónico para la escritura en una segunda lengua, quienes estudian el rol que juega el feedback mediado por computador en diferentes áreas de investigación e instrucción.

Este artículo comienza con un análisis crítico acerca de la problemática del FCE en los últimos quince años y los supuestos teóricos que sustentan diferentes puntos de vista sobre la importancia y efectividad que el FCE tiene en la adquisición de una segunda lengua. Posteriormente, se presenta el modelo teórico de investigación en FCE, que se sustenta en tres líneas de investigación, a saber, la ASL, la producción escrita en una $L 2$ y el aprendizaje de lenguas asistido por computador. Hacia el final del artículo, se comenta acerca de cómo estudios en esta área de interés, en los últimos años, han obtenido resultados relevantes en torno al papel que tiene el feedback correctivo en la ASL y se entregan proyecciones para los profesores, estudiantes e investigadores de segundas lenguas de cómo distintas formas de feedback son capaces de contribuir al desarrollo de la escritura en una L2.

\section{Fundamentos teóricos}

\subsection{La controversia en torno al feedback correctivo escrito}

La habilidad de producción escrita es una de las más complejas que enfrenta en su desarroIlo un estudiante de L2; consecuentemente, los profesores son Ilamados a monitorear este proceso ayudándolos a mejorar, entre otras cosas, la proficiencia. Generalmente, la superación de la ocurrencia de errores gramaticales depende de la forma en que son corregidos y por lo tanto el feedback correctivo escrito es un aspecto esencial en cualquier curso de escritura en una segunda lengua. Hyland (2003) afirma que una de las mayores preocupaciones de los escritores de segundas lenguas es la ausencia de errores en su trabajo escrito ya que éste es evaluado en un contexto académico en el que la precisión gramatical es un tema importante.

Sin embargo, esto que para muchos investigadores, profesores y estudiantes de L2 pareciera ser un componente fundamental en el proceso de adquisición y aprendizaje de una segunda lengua no ha estado exento de controversia. Hay un hito en la literatura que sin duda ha sido el que ha impulsado la investigación de la última década y me refiero a la discusión académica entre Truscott y Ferris a mediados de los años 90 (Truscott, 1996, 2004, 2007; Ferris, 1999, 2004, 2010). En 1996 Truscott en su artículo seminal "The Case Against Grammar Correction in L2 Writing Classes" declaró que la entrega de feedback correctivo escrito a los estudiantes de inglés como segunda lengua no era efectivo y que además podía ser dañino para el desarrollo de la escritura (Milton, 2006; Bitchener, 2008; Bitchener y Knoch, 2008; Ellis y otros, 2008; Sheen, 2011):

(a) Substantial research shows it to be ineffective and none shows it to be helpful in any interesting sense; (b) for both theoretical and practical reasons, one can expect it to be ineffective; and (c) it has harmful effects.

Ferris (1999) está de acuerdo con algunos 
de estos puntos; sin embargo, argumenta que el uso del FC es necesario porque los estudiantes quieren ser corregidos, los profesores exigen a sus estudiantes precisión gramatical en sus textos escritos y sostiene que los estudiantes necesitan desarrollar la habilidad de autoedición de sus errores.

A pesar del debate entre Ferris y Truscott en relación a la efectividad de la corrección de los errores en textos escritos, existe un punto en el que estos autores sí están de acuerdo, a saber, que la investigación en la corrección de los errores en la producción escrita en una L2 es insuficiente, poco concluyente y que faltan estudios con diseños metodológicos robustos.

A la luz de los resultados de la investigación que ha estudiado el FCE, no es sorprendente el hecho de que la corrección de los errores escritos sea polémica; la revisión literaria relacionada con esta controversia reafirma esta aseveración, debido a que es difícil obtener conclusiones claras debido a que los resultados se han obtenido de una variedad de poblaciones, tratamientos, diseños de investigación y enfoques hacia esta problemática (Hyland y Hyland, 2006; Ellis y otros, 2008). Ambas posturas se sustentan en fundamentos teóricos que han dominado la adquisición de una L2 en los últimos 40 años.

\subsection{Uso del FC en la enseñanza de una $L 2$}

En la década de los 70 la adquisición de segundas lenguas emergió fuertemente como campo de estudio, y en la década posterior varios enfoques comenzaron a desarrollarse en paraleIo. La Gramática Universal (Chomsky, 1986; Cook, 1988) es la primera gran teoría a la que refieren varios modelos de aprendizaje y adquisición de segundas lenguas. Aunque esta teoría no está principalmente enfocada en la ASL, su influencia en este proceso ha sido reconocida como importante. La GU afirma que determinados principios comunes subyacen a todas las lenguas naturales y en ella se sustenta que estos principios son innatos dentro de nuestra condición humana, existen por lo tanto una serie de reglas que ayudan a los niños a adquirir su lengua materna; entonces, el conocimiento de la lengua es inconsciente, no se aprende sino que se deriva de la GU (Sheen, 2011). Basados en las ideas innatistas y en aquellas que promueven la similitud de los procesos de adquisición de la $L 1$ y la $L 2$ es que algunos investigadores (Krashen, 1981, 1982) han sido defensores de un enfoque natural hacia la enseñanza de la $L$ 2. Se piensa que tener acceso a una gran cantidad de estímulo comprensible es una condición necesaria y suficiente para la adquisición de la L2 (Van Beuningen, 2010); para ello, se espera que los aprendientes comprendan el input disponible haciendo referencia a su significado sobre la base de la información lingüística subyacente al contexto comunicativo; como resultado, la competencia gramatical debería emerger automáticamente sin necesidad alguna de evidencia negativa (Van Beuningen, 2010; Sheen, 2011). Hoy en día, sin embargo, existe gran consenso de que el proceso de adquisición de la L1 y la L2 obedece a fenómenos cognitivos distintos; además, existe evidencia suficiente para sugerir que el input comprensible no es una condición suficiente para desarrollar un nivel de precisión gramatical similar al de un hablante nativo; por otra parte, si solamente las formas adquiridas pueden llevar a la producción de habla espontánea, entonces sería imposible aprender una segunda lengua o lengua extranjera en la sala de clases. Entonces, alguna atención a las formas lingüísticas sí es necesaria para que los hablantes de L2 progresen en la precisión gramatical; de esta manera, los enfoques y metodologías comunicativas actuales incorporan la instrucción de la gramática. En este nuevo escenario, la evidencia negativa y por consiguiente el feedback correctivo son un factor potencial en el desarrollo del conocimiento explícito, que resulta fundamental para generar conocimiento implícito, es decir, adquisición. Los investigadores han agregado otros conceptos que han complementado la teoría de Krashen, como la atención, la comprensión, la negociación del significado, 
la interacción que tiene lugar entre hablantes para asegurar un entendimiento común de los significados intercambiados en el discurso, que según Ellis (1997) tienen un papel importante en el proceso de adquisición también. A partir de lo anterior, Long (1996) propone lo que se conoce en ASL como la hipótesis de la interacción, en la que el input y el output son necesarios para la adquisición de una segunda lengua. Para tener mayor entendimiento de cómo esto funciona, se debería poner mayor atención a las interacciones con las que los hablantes se comunican. Para Long (1996), estas interacciones no son solamente una fuente del input lingüístico, sino que son consideradas como intercambios que permiten a las partes negociar el significado del input y esta negociación tiene como resultado cambios en la complejidad del input. De esta manera, cuando los hablantes se comunican, emiten e interpretan señales propias, así como también de la comprensión de su interlocutor, de modo que se producen ajustes de contenido, estructura conversacional y de forma lingüística, hasta que se alcanza un nivel de entendimiento aceptable (Long, 1996). Es en este contexto, el de la negociación del significado, que la noción de focalización en la forma (FF) acuñada por Long (1991) se transforma en una intervención instruccional potente, que atrae la atención de los estudiantes a los elementos lingüísticos que surgen incidentalmente en lecciones en las que el foco está dado por el significado o la comunicación. Este punto es de vital importancia para entender el papel que juega la FF en la adquisición de una L2, porque los investigadores concuerdan en que el objetivo fundamental en la sala de clases es enseñar la lengua para la comunicación, por consiguiente el contexto de su aplicación es el acto de la comunicación y el desarrollo de la competencia comunicativa (Doughty y Williams, 1998); el interés de los investigadores se centra en fa- cilitar el despliegue de formas lingüísticas para conseguir mejorar los procesos comunicativos. Una de las herramientas que se identifican como un instrumento potencial de la focalización en la forma es la corrección de los errores (Ellis, 2005). Consecuentemente, el feedback correctivo es una metodología reactiva de la focalización en la forma que tiene la particularidad de centrar la atención del aprendiente en las estructuras lingüísticas en el contexto de realización de una tarea comunicativa de una manera personalizada e individual (Van Beuningen, 2010).

Una segunda perspectiva fundamental de la metodología de focalización en la forma se encuentra en la hipótesis de "noticing" (Schmidt, 1990, 2001). El concepto de "noticing" (notar) combina las dos nociones lingüísticas cognitivas cruciales de atención y conciencia, en el sentido de darse cuenta de algún fenómeno en particular (Sheen, 2011). Esta hipótesis establece que la adquisición subliminal de una L2 es imposible (Van Beuningen, 2010), y que sólo a través de la atención consciente el input se puede convertir en intake ${ }^{1}$. Así Schmidt (1990) argumenta que darse cuenta es una condición necesaria para el aprendizaje de lenguas; este investigador piensa que el que aprende se da cuenta de la diferencia que existe entre lo que puede producir y lo que necesita producir, entre lo que produce y lo que los hablantes nativos producen. Cuando la atención hacia las formas lingüísticas es considerada facilitadora para el desarrollo de la interlengua, las intervenciones de focalización en la forma como el FC promueven el proceso de adquisición de segundas lenguas, les permiten a los estudiantes notar la diferencia entre el output de su propia interlengua y el input de la segunda lengua (Van Beuningen, 2010). En este sentido, el feedback correctivo escrito muestra ventajas sobre el feedback correctivo oral, debido a que, aunque ambos brindan oportunidades de con-

1 Se entiende por intake el input comprensivo que impacta en el sistema lingüístico en desarrollo del sujeto que aprende (Sheen, 2011). 
ciencia de las diferencias entre los sistemas de interlengua y lengua meta (Ferris, 2010; Sheen, 2011), es probable que los aprendientes no siempre sean capaces de realizar la comparación cognitiva en línea que la lengua oral utiliza. La producción oral en línea y la entrega de FC pueden llegar a ser muy exigentes y generar una carga cognitiva excesiva; en cambio, en la escritura, los estudiantes tienen más tiempo para comparar su output con el FC que reciben, lo que aumenta la probabilidad de notar los vacíos en su interlengua (Ferris, 2010; Van Beuningen, 2010; Sheen, 2011)

Un último supuesto teórico que sustenta la provisión de feedback correctivo es el que se refiere al output (Swain, 1985). Durante el auge del enfoque comunicativo basado en la GU y las teorías de Krashen, el output era un producto, un resultado, y no un factor del proceso de adquisición de una segunda lengua. Swain (1985) argumenta que la importancia del output en la L2 radica en el hecho de que la producción de éste impulsa al estudiante a procesar la lengua con mayor profundidad y con mayor esfuerzo mental que el realizado durante la comprensión lectora y la comprensión auditiva. Los puntos que sustentan esta hipótesis se pueden resumir de la siguiente forma (Van Beuningen, 2010; Sheen, 2011):

a) El output puede gatillar el tipo de reflexión metalingüística que es fundamental para el desarrollo de la interlengua.

b) Es un medio para probar hipótesis de formas lingüísticas específicas

c) Tiene la habilidad para que los estudiantes se den cuenta de los vacíos y problemas existentes en su interlengua.

Una vez más, se puede argumentar que los efectos asociados con el output escrito y el feedback correctivo superan a los de los usos de la lengua oral y de su corrección, debido a que en el proceso de escritura los estudiantes no tienen las mismas restricciones de tiempo que aquellas existentes en la comunicación oral en línea y, por lo tanto, es factible que el resultado del output en combinación con el FC sea beneficioso.

\subsection{Oposición al uso del FC en la enseñanza de una L2}

Mientras algunos investigadores han impulsado el uso del FC argumentando que es un elemento facilitador de la adquisición de una segunda lengua, otros han sostenido en el tiempo que la corrección de los errores es completamente innecesaria, ineficiente, y hasta la han calificado de dañina para este proceso (Krashen, 1985; Truscott, 1996, 1999). En el centro del debate todavía se encuentra el argumento consistente de Truscott $(2004,2007)$ de que el feedback correctivo, especialmente el escrito, no es efectivo para promover la adquisición y el aprendizaje de una L2. Este punto de vista se sostiene en argumentos prácticos y teóricos, siendo uno de los principales el rol del conocimiento del sistema gramatical de la segunda lengua, lo que ha sido acuñado como conocimiento explícito, declarativo, en oposición al conocimiento implícito, procedimental (Krashen, 1981; DeKeyser, 1998, 2003). El conocimiento explícito implica conciencia de las reglas gramaticales; el conocimiento implícito, por otra parte, es inconsciente, de fácil acceso durante la comunicación en línea. En la actualidad se asume que este último conocimiento es el que permite al estudiante comunicarse espontáneamente. Por consiguiente, los adherentes de esta postura desconocen el valor que tiene el conocimiento explícito en sí mismo, así como también la conexión que existe entre conocimiento explícito e implícito, y por lo tanto argumentan que si el FC es capaz de generar algún tipo de conocimiento, éste solamente puede ser de naturaleza explícita, pero no implícita. De este modo, los mecanismos internos pueden ser activados solamente por medio de la evidencia positiva, y no mediante la evidencia negativa. Schwartz (1986) reconoce que la evidencia negativa puede resultar en adquisición del conocimiento explícito de la gramática, pero argumenta que no existe 
un mecanismo que pueda transferir este conocimiento a conocimiento implícito. Adicionalmente, Krashen (1981) argumenta que los aprendientes pueden solamente utilizar su conocimiento explícito durante la fase de edición de su output después que ha sido iniciado por el sistema de adquisición; en palabras de Krashen, "durante la fase del monitoreo".

Otros académicos (Doughty y Williams, 1998), sin embargo, creen que el conocimiento explícito alimenta al implícito, ayudando al que aprende a darse cuenta de las estructuras formales del input. Desde esta perspectiva se espera que el FC promueva el desarrollo de la interlengua porque facilita el proceso de ver la diferencia o vacío entre la interlengua y la lengua meta. Otra objeción teórica utilizada por Truscott (1996) en su caso en contra de la corrección de los errores se fundamenta en la hipótesis del "orden natural" de Krashen (1981), que se refiere a un presunto orden en el cual naturalmente los seres humanos vamos incorporando la información gramatical en el proceso de adquisición de una segunda lengua sin necesidad de que haya un programa con un orden preestablecido de instrucción de estructuras. Pienemann (1989) sugiere que los aprendientes adquieren sólo las estructuras para las que están preparados; aquellos rasgos gramaticales que están más allá del estado de desarrollo del aprendiente no son enseñables porque el proceso de adquisición no puede ser modelado de acuerdo a los requerimientos de la instrucción formal (Pienemann, 1989). Según estos teóricos, para que el FC tenga algún efecto positivo, éste debiera estar alineado con el nivel de desarrollo de los aprendientes de la L2, de lo contrario las estructuras que se desean corregir no van a estar disponibles para su adquisición; por consiguiente concluyen que la entrega de un FC alineado al nivel de desarrollo lingüístico de los estudiantes no es un objetivo factible de lograry, en consecuencia, la corrección de los errores no puede ser beneficiosa para la ASL (Truscott, 1996). Sin embargo, es de vital importancia señalar que todavía las ideas y el conocimiento relacionado con las secuencias de desarrollo son limitados para ser utilizados en la enseñanza y aprendizaje de segundas lenguas (Ellis, 1997; Van Beuningen, 2010).

Un último argumento expresado por los teóricos que se oponen a la entrega de FC (Truscott, 1996) es que el FC puede ser contraproductivo e incluso dañino para el proceso de adquisición de una L2. Contraproductivo porque los profesores y los estudiantes pueden estar mucho tiempo corrigiendo en vez de desarrollar actividades de práctica adicional, y dañino porque el tomar conciencia de los errores conduce a stress y ansiedad, en palabras de Krashen (1981) afecta el filtro afectivo, especialmente cuando se comete el mismo error; como resultado, los estudiantes evitan las formas erróneas en los futuros textos escritos. El resultado, según estos teóricos, es una escritura simplificada, donde el estudiante tiene mayor control sobre los elementos más estables de su interlengua, pero evitando la ampliación de su repertorio.

\subsection{Orientaciones en torno al feedback en la producción escrita en una L2}

A pesar de las críticas al feedback correctivo, la investigación en ASL insiste en que la optimización de los procesos de adquisición se produce cuando los estudiantes se focalizan en el significado y en la forma (Doughty y Varela, 1998). Sin embargo, en la práctica, existen problemas para la entrega de un feedback correctivo basado en el enfoque en la forma porque muchos profesores y estudiantes de lengua están abocados, por diversas presiones sociales, a pasar una gran cantidad de tiempo en tratar de erradicar todos los errores de interlengua en el nivel oracional. Muchas estrategias han sido sindicadas para ayudar a los profesores en esta tarea (Ferris, 2002) y hacer el feedback correctivo más efectivo. Sin embargo, las presiones de tiempo, el número de estudiantes hacen que muchas veces los profesores fallen en el objetivo de entregar feedback correctivo apropiado (Milton, 2006). La sistematicidad que han alcanzado los estudios 
recientes en ASL y en producción escrita permite tener una perspectiva futura interesante y optimista al respecto, aunque, como se ha señalado con anterioridad, mayor investigación es necesaria para obtener resultados contundentes. De acuerdo a lo anterior, y considerando algunos estudios recientes, se estima que la investigación en feedback correctivo escrito mediado por computador puede ser una gran contribución en la búsqueda de la efectividad del feedback, porque hoy en día existe un amplio desarrollo educacional de las tecnologías de información y comunicación y el feedback electrónico no está al margen de este fenómeno. Los estudiantes que están conectados a una pantalla están propensos a recibir algún tipo de respuesta; ese feedback puede venir como una reacción a través de un e-mail, un puntaje asignado por un programa automatizado, comentarios realizados por algún par o un tutor experto que ayude a generar una revisión final de un texto (Ware y Warschauer, 2006).

Para los profesores que ven a la escritura como el dominio de un compendio de destrezas, el feedback electrónico sirve entonces como información automatizada proporcionada por una computadora. Sistemas sofisticados de software que generan una respuesta inmediata de evaluación sobre la escritura del estudiante están disponibles hoy en el mercado, y para aquellos que ven a la escritura como una práctica social, el término electrónico indica el medio por el cual se proporciona información humana.

Aun cuando la investigación de la eficacia del feedback mediado por computador es todavía un tanto limitada, principalmente por el ritmo acelerado de la evolución de la tecnología, que puede hacer que los resultados rápidamente sean obsoletos, estudios recientes sugieren que estos ambientes tecnológicos que promueven el FC favorecen la reflexión y el desarrollo de una segunda lengua. Estos hallazgos son alentadores, pero todavía poco se conoce acerca de cómo el feedback mediado por computador difiere del tradicional y si las mismas variables que dan cuenta de la adquisición en este último ambiente son cruciales para el aprendizaje en el contexto de la comunicación mediada por computador (Loewen y Erlam, 2006; Hyland, 2010; Van Beuningen, 2010).

Como el rol del FC escrito en la adquisición aún es controversial, tanto desde el punto de vista práctico como también desde el teórico, el tema es atractivo para los investigadores que buscan respuesta a la pregunta de si el FC y en especial el escrito es beneficioso o no para la adquisición de una L2. Ferris (2004) reconoce que los estudios de FCE aún están lejos de proveer evidencia concluyente acerca de los beneficios; sin embargo, seis años más tarde afirma que los diseños empíricos claros y sólidos basados en el paradigma de ASL son impresionantes, con resultados favorables hacia la corrección de los errores, y se pregunta si la aplicación de los hallazgos es factible en la enseñanza de la producción escrita en una L2 (Ferris, 2010). A partir de aquella reflexión y del aumento vertiginoso que ha tenido la investigación en el rol que tiene la tecnología en el aprendizaje de una segunda lengua, se propone un modelo de investigación para estudiar el FCE.

\section{Modelo de investigación en feedback correctivo escrito}

Basado en lo propuesto por Ferris (2010) en relación a una posible intersección en metodologías de investigación entre ASL y escritura en una segunda lengua, más la inminente importancia que tiene el uso de la tecnología en el aprendizaje de una L2 (Hyland, 2010; Sheen, 2011), el modelo de investigación en feedback correctivo escrito que se propone en este artículo se sustenta en tres ejes que se complementan entre sí, a saber, la rigurosidad metodológica que aportan los estudios de FCE desde la perspectiva de la ASL, la practibilidad y pertinencia de los estudios de FCE que promueven el desarrollo de habilidades escritas tales como la revisión y la edición tex- 
tual en una L2 en contextos de aprendizaje real y el rol que ha tenido la tecnología en los últimos veinte años, específicamente en cómo las innovaciones en esta área pueden impactar en el feedback y especialmente en el feedback correctivo en el aprendizaje de una $\mathrm{L} 2$.

\subsection{La rigurosidad de la investigación en feedback correctivo escrito desde la perspectiva de la adquisición de segundas lenguas}

Sin lugar a dudas, la importancia de la polémica en torno al FC escrito es que, previamente a la demanda realizada por Truscott (1996) acerca de la inefectividad del feedback correctivo, nadie había discutido el supuesto ampliamente difundido acerca de la eficacia de corregir los errores escritos de los hablantes de una segunda lengua. Por consiguiente, la evidencia empírica existente era bastante exigua y sólo se limitaba a algunos estudios que se concentraban en una cuestión de eficacia y en el diseño de investigación (Bitchener, 2008), y aunque ya ha pasado más de una década desde la controversia, la literatura indica que poco a poco se han realizado estudios con un diseño de investigación robusto y que examinan la efectividad del FC escrito en el tiempo. Estos estudios se concentran en el potencial que tiene el feedback correctivo escrito para ayudar al desarrollo de la interlengua de los hablantes, que surge del concepto de escribir para aprender (Van Beuningen, 2010) y que se basa fuertemente en el paradigma psicolingüístico y cognitivo de la ASL. Las condiciones experimentales para su realización han sido bien controladas y los efectos del FC comparados con el desempeño en precisión gramatical de los estudiantes.

Bitchener (2008) ha revisado un gran número de estudios acerca de los efectos del feedback correctivo escrito, señalando que sólo algunos de ellos han incluido un grupo control, por lo que no ha sido posible comparar entre aquellos estudiantes que reciben y los que no reciben $\mathrm{FC}$; por esta razón, aquellos estudios que no comparan los efectos del FC no entregan evidencia de su efectividad, y los logros de los estudiantes pueden ser el resultado de factores independientes del FC. Un grupo control, como indican los teóricos (Bitchener, 2008; Ellis y otros, 2008), entonces, es esencial para demostrar la efectividad del feedback correctivo escrito; en consecuencia, los hallazgos empíricos fundamentales se encontrarán en aquellos estudios que lo incorporan en el diseño metodológico. Bitchener considera además que las investigaciones en FCE revisadas tenían otras falencias metodológicas; por ejemplo, no fueron capaces de demostrar que las ganancias resultantes del FC podían ser aplicadas a la producción escrita de textos nuevos, sólo arrojaron evidencia de que el FC es capaz de ayudar a los estudiantes a mejorar la precisión gramatical en un nuevo borrador de un texto que es una revisión del texto original. No obstante lo anterior, también es oportuno considerar lo siguiente: varios de los estudios mencionados por Bitchener (2008) han entregado información importante para las prácticas académicas cotidianas y se han focalizado en evaluar la eficacia de un enfoque en particular del FC capaz de desarrollar estrategias de edición en los estudiantes.

El enfoque principal de los estudios que han investigado la eficacia de los diferentes tipos de FCE ha sido el grado en que la información directa o indirecta facilita el mejoramiento de la precisión gramatical. Aunque estos términos no siempre se han utilizado constantemente en la literatura, el feedback correctivo directo (FCD) puede ser definido como la provisión de la forma correcta o la estructura lingüística por encima o cerca del error lingüístico (Ferris, 2003; Bitchener, Young y Cameron, 2005; Sheen, 2011). Este puede incluir el tachar una palabra, frase o morfema que no sea necesario; la inserción de una palabra, frase o morfema, o la provisión de la forma correcta (Bitchener, 2008). Otras formas de feedback directo (FD) pueden ser una explicación metalingüística escrita² o una explicación metalingüística oral³. Por otra parte, el feedback correctivo indirecto $(\mathrm{FCl})$ indica que, de alguna 
manera, un error ha ocurrido. Este puede ser proporcionado en una de las siguientes maneras: subrayando el error, registrando al margen la cantidad de errores en una línea determinada o utilizando un código para mostrar que el error ha ocurrido y qué tipo de error es (Ellis, 2009; Sheen, 2011); son los estudiantes quienes deben resolver y corregir el problema en el cual se ha focalizado su atención.

Bitchener (2008) menciona que existen pocos estudios en FCE que comparen resultados entre $\mathrm{FCD}$ y $\mathrm{FCl}$, y que en estudios preliminares hubo inclinación por el segundo debido a la capacidad que genera en los estudiantes el reflexionar acerca de los errores, lo que puede promover la adquisición a largo plazo. Algunos teóricos, sin embargo, que no desmerecen el valor del FCl estiman que el FCD es preferido por profesores y alumnos, además sugieren que el FCD reduce el tipo de confusión que resulta cuando los estudiantes no logran comprender o recordar los códigos utilizados por el profesor. Bitchener (2008) sostiene que, analizando ventajas y desventajas, no es posible determinar cuál de los dos tipos de feedback es mejor. Para la construcción del modelo teórico de este artículo, se estima que el FCD tiene ventajas por sobre el $\mathrm{FCl}$, basado en que, así como la evidencia del feedback correctivo oral (FCO) sostiene que la información explícita del feedback correctivo facilita el conocimiento implícito, del mismo modo la información del conocimiento explícito que entrega el FCD a través de la focalización del error y las diversas formas de información metalingüística también ayuda a los estudiantes a desarrollar conocimiento implícito y de esta forma generar entendimiento (Schmidt, 1994; Sheen, 2011).

Otra distinción relevante en función de la rigurosidad metodológica es la distinción entre feedback correctivo focalizado (FCF) y feedback correctivo no focalizado (FCNF). Este último corresponde a la práctica habitual de los profesores de escritura, quienes corrigen todos los errores en los trabajos escritos de los estudiantes (Ellis y otros, 2008), en contraste con el FC focalizado, que selecciona errores específicos para ser corregidos y descarta otros. Un FC altamente focalizado se va a concentrar en una, a lo más dos categorías. De alguna manera un feedback correctivo intermedio se puede concentrar en más de dos categorías, pero siempre restringiéndose a un grupo limitado de ellas. Según Ellis y otros (2008), existen razones teóricas sólidas que avalan el argumento de que el FC focalizado puede ser más acertado que el FC no focalizado. Los estudiantes son más propicios a poner atención a correcciones dirigidas a uno o a un número limitado de tipos de errores y más propensos a desarrollar mayor comprensión de la naturaleza del error y la corrección necesaria. Si la atención (noticing) y el entendimiento (Schmidt, 1994) son necesarios para la adquisición, entonces el FC focalizado está claramente en mejores condiciones para producir mejores resultados. La evidencia obtenida por los investigadores (Sheen, 2007; Bitchener y Knoch, 2008) da cuenta de la eficacia del FC focalizado.

Un último alcance en relación a un diseño metodológico consistente y robusto es la medición de la retención a través del tiempo; se debe incorporar en el diseño metodológico una forma de evaluación diferida. Al hacerlo, sin embargo, no es posible eliminar el efecto de las variables que intervienen entre el postest y un postest diferido (por ejemplo, los estudiantes pueden acceder a más instrucción o someterse a la práctica más allá del tiempo de clase); de todas maneras, se puede utilizar este test diferido para medir el nivel de retención que se observó en el postest. Esto es fundamental para ver si el feedback co-

2 La provisión de reglas gramaticales y los ejemplos al final de la escritura de un estudiante con una referencia a los lugares en el texto donde él ha cometido un error (Sheen, 2011).

3 Una minilección en la que las reglas y ejemplos se presentan, se practican y se discuten, en una modalidad profesor-alumno o profesor con grupos pequeños de alumnos (Bitchener, 2008) 
rrectivo tiene efectos en la internalización de las estructuras en el mediano y largo plazo, de manera que los estudiantes comprueben que han superado la etapa del error en la generación de textos nuevos y así tener una sensación de avance en su competencia lingüística en el desarrollo de la habilidad escrita.

\subsection{El rol del feedback correctivo en el proceso de producción escrita en una L2}

La enseñanza de la producción escrita en una segunda lengua ha sido influenciada históricamente por las tendencias teóricas que han determinado la instrucción en una segunda lengua. En una primera etapa, un enfoque estructuralista considera a la escritura en una segunda lengua como una representación ortográfica de los rasgos léxicos y sintácticos del habla de la L2 (Sheen, 2011); el desarrollo de las habilidades escritas involucra la producción de patrones gramaticales correctos en ejercicios controlados diseñados para minimizar los errores; el feedback correctivo se entrega en aquellos casos en que se produce un error. En etapas posteriores al estructuralismo, la escritura no era considerada en el proceso de enseñanza de la lengua y posteriormente fue fuertemente influenciada por el proceso pedagógico de la L1 y las teorías de adquisición de Krashen (1982). Esta visión de la escritura en una segunda lengua, en contraposición al enfoque estructuralista, minimiza el rol de la corrección del error escrito en las clases de escritura y prioriza el proceso y la organización; sin embargo, esto último no ha estado exento de crítica, ya que algunos proponentes sostienen que este enfoque, que se basa fuertemente en las teorías de escritura de la lengua materna, no puede ser aplicado íntegramente a la producción escrita en una segunda lengua ya que ambos procesos difieren fuertemente el uno del otro (Sheen, 2011)

La investigación del feedback correctivo post proceso de escritura ha tratado de evitar los excesos del pasado en torno a la obsesión por los errores, pero no rechaza la temática de la preci- sión gramatical y la corrección de los errores de manera contextualizada dentro de un proceso de escritura recursivo, que prioriza la focalización de patrones frecuentes de errores escritos y que busca desarrollar habilidades de edición del trabajo escrito con el FCE posicionado como un apoyo fundamental para obtener este objetivo (Ferris, 2010). Estos estudios han sido realizados en el contexto de la sala de clase y sus diseños pueden o no contar con un diseño de investigación que incorpore pretest-postest y un grupo control; algunos estudios no definen qué tipo de errores escritos reciben feedback correctivo y existe una gran variación en cómo y por quién es entregado el FC. Esta falta de rigor empírico ha sido objeto de crítica por parte de los investigadores de ASL; sin embargo, ha sido justificada en función de la practicabilidad en su aplicación en contextos educacionales reales. Aún más, en su argumentación, los investigadores de escritura cuestionan el hecho de que la investigación en ASL está tan abocada al rigor empírico y a la focalización en algunos rasgos lingüísticos que es muy difícil transferir sus hallazgos a una situación de clase real (Ferris, 2010). En consecuencia, los estudios en escritura de una L2 buscan el desarrollo de estrategias de escritura efectivas y que los procesos impacten la escritura de los estudiantes en el futuro, que es uno de los principales objetivos de la enseñanza de una L2; por lo tanto, partiendo de la pregunta de si el FC ayuda a los estudiantes de escritura, los estudios de revisión, reformulación y otro tipo entregan información a los profesores y a los investigadores para refinar sus prácticas. Pero también se argumenta que la revisión y edición de un texto específico después de recibir FC puede ser un paso intermedio entre el feedback experto acerca de un rasgo específico y su adquisición a largo plazo (Ferris, 2004, 2006).

\subsection{El uso de la tecnología en el feedback correctivo escrito}

La utilización de la tecnología en el aprendizaje de idiomas y, más concretamente, cómo 
las innovaciones en esta área pueden tener un impacto en el feedback ha generado un cuerpo creciente de investigación conducente a evidencia empírica acerca de su efectividad en esta modalidad. En los estudios se destaca que el feedback electrónico se refiere a los medios por los cuales se puede entregar información a través de la tecnología. Para los investigadores, la cuestión central es cómo la respuesta mediada por computador podría imitar o incluso mejorar los resultados informados por la investigación acerca de la interacción de pares en un contexto presencial. Algunos estudios han comparado las prácticas conocidas en el aula con las actividades realizadas con o a través del medio electrónico (Ware y Warschauer, 2006).

Pellettieri (2000) dio muestras de una mayor atención a la forma, a la negociación de significado, y la modificación lingüística en la escritura en línea. DiGiovanni y Nagaswami (2001) demostraron que los estudiantes estaban más centrados en la tarea al proporcionar feedback durante tiempo real; los instructores señalaron las ventajas del medio electrónico, que incluye la capacidad de monitorear las conversaciones entre pares e imprimir las transcripciones. Ellos sugieren un efecto indirecto positivo para los estudiantes, que pueden sentir que este tipo de supervisión docente es adecuada. En materia de sistemas tutoriales inteligentes (STI) para el español como LE se han realizado estudios empíricos centrados en estrategias de feedback correctivo comunicativo (Ferreira, 2003, 2006, 2007). Las estrategias correctivas que se estudiaron fueron del tipo Prompting-Answer-Strategies (PAS), aquellas que inducen a la autorreparación del error, es decir, estrategias que intentan extraer o elicitar la respuesta correcta por parte del alumno; y las estrategias del tipo GivenAnswer-Strategies (GAS), aquellas en las que se tiende a dar la respuesta correcta o corregir de manera explícita el error por parte del profesor o tutor (Ferreira, 2003, 2006, 2007). Se han determinado los tipos, frecuencias y efectividad de estrategias de feedback orales utilizadas en la enseñanza del español como lengua extranjera en diferentes contextos de aprendizaje de lengua: un estudio observacional (Ferreira, 2006) de interacciones profesor-alumno en clases tradicionales presenciales (modalidad face-to-face), un estudio de caso de interacciones en clases tutoriales presenciales (modalidad one-to-one) (Ferreira, Moore y Mellish, 2007) y estudios experimentales (Ferreira, 2007) en los cuales los estudiantes interactuaban con una aplicación computacional "no presencial" (modalidad oneto-one a distancia) accesada en línea a través de la red internet. Los resultados de estos estudios permitieron definir un modelo de tratamiento de errores gramaticales y estrategias de feedback correctivo oral (Ferreira, 2003; Ferreira, Moore y Mellish, 2007; Ferreira y Atkinson, 2009). Sobre la base de dichos resultados se ha propuesto que un STI para la enseñanza del español como LE en un contexto gramatical comunicativo debería implementar estrategias de feedback correctivo que estimulen a los estudiantes a la autorreparación de sus errores, esto es, claves metalingüísticas, elicitaciones y clarificaciones lingüísticas (Ferreira, 2003, 2006, 2007). Más recientemente, se ha propuesto una arquitectura para el desarrollo de un STI para el español como LE, el sistema ELE-TUTOR (Ferreira y otros, 2012). Este sistema tiene por objetivo mejorar la precisión gramatical de alumnos de nivel B2 en actividades comunicativas y cuenta por el momento de manera prototípica con un parser que procesa errores de los contenidos de futuro simple y compuesto, condicional simple y compuesto y estilo indirecto correspondientes a contenidos gramaticales propios del nivel B2 (Ferreira y Kotz, 2010; Ferreira y otros, 2012; Kotz y Ferreira, 2013). Además, se ha propuesto un modelo de estudiante (Barrientos, Ferreira y Salcedo, 2012) que considera el nivel de proficiencia del alumno en la lengua extranjera como criterio de selección de las estrategias de retroalimentación, ya que diversos estudios evidencian que cada nivel de competencia requiere 
de estrategias diferentes (Ferreira, 2007). Otro aspecto que se tuvo en cuenta en el diseño del modelo del estudiante es el estilo de aprendizaje del alumno (Sawyer y Ranta, 2001). Los estudiantes con un estilo de aprendizaje más holístico se benefician más de un feedback de elicitación, mientras que estudiantes que se inclinan por un procesamiento más analítico prefieren un feedback con claves metalingüísticas. Con el fin de favorecer a ambos grupos se postula un diseño que combine ambos tipos de feedback (Ferreira y otros, 2012).

En síntesis, de acuerdo a los estudios mencionados anteriormente, Ware y Warschauer (2006) identifican algunas líneas de investigación en la temática del feedback electrónico y la escritura de segundas lenguas capaces de clasificar las investigaciones en dos grandes grupos: estudios de feedback automático que se centran en los aspectos individuales de la escritura, que han promovido especialmente el desarrollo comercial de sistemas de evaluación, y estudios de feedback humano mediado por computador. En esta segunda línea de investigación se aborda la integración de la tecnología en la sala de clases y en qué medida se explota el potencial social e interactivo que ella conlleva. Se destaca entonces cómo el feedback electrónico puede ser entregado a través de la tecnología, simulando las condiciones que los pares y expertos pudieran enfrentar en una situación de clase presencial. Existe un tercer grupo que está relacionado con una línea de investigación que aborda la temática de cómo la tecnología es integrada al currículo y cómo los profesores la integran como un recurso más en función del desarrollo de la habilidad escrita.

Para la elaboración del modelo teórico que se presenta en este artículo se elige el feedback correctivo mediado por computador, debido a la característica de su aplicación en ambientes presenciales como también en ambientes no presenciales, lo que se considera una condición óptima para ser utilizada en el proceso de escritura en una segunda lengua.

\subsection{Operacionalización de un modelo de investigación para el tratamiento de los errores a través de FCE}

En el modelo propuesto, se ha tratado de evitar las falencias de diseño a las que ha aludido Truscott (2007) y se ha incorporado e integrado lo aportado por cada una de las líneas de investigación antes referidas, comenzando por el aporte de los estudios de FCE desde la perspectiva de la ASL, de los estudios de FCE que promueven el desarrollo de habilidades escritas tales como la revisión y la edición textual, y de los estudios acerca de las innovaciones tecnológicas y su impacto en el feedback correctivo en el aprendizaje de una L2.

Como se puede ver en la figura 1, el modelo contempla un pretest, un postest inmediato y un postest diferido para los grupos experimentales y el grupo control. Un ciclo correspondiente al tratamiento, que comienza con una tarea escrita para ambos grupos; el tratamiento de feedback correctivo escrito propiamente tal para los grupos experimentales y la entrega de comentarios generales del texto escrito para el grupo control; el proceso de revisión y edición del mismo texto escrito para ambos grupos; una segunda ronda de tratamiento de feedback correctivo para los grupos experimentales y de comentarios generales para el grupo control. Este ciclo puede ser repetido, comenzando con una nueva tarea escrita, las veces que el investigador lo determine necesario de acuerdo a los propósitos del estudio y al contexto de aplicación.

El modelo promueve un análisis focalizado de los errores. La dicotomía FC focalizado - no focalizado se refiere a la amplitud de acción de las metodologías de corrección de los errores. La no focalizada involucra la corrección de todos los errores de escritura independientemente de su categoría; la focalizada corrige rasgos lingüísticos específicos solamente $y$, por consiguiente, los errores que están fuera del dominio de corrección no son tratados. En este aspecto, el modelo se sustenta en Ellis y otros (2008), 
quienes declaran que los estudiantes están más propensos a notar sus errores y a comprender las correcciones cuando se especifica un grupo reducido de ellos; por otra parte, Bitchener (2008) argumenta que el feedback correctivo no focalizado no es el método más eficiente, porque los hablantes tienen una capacidad limitada de procesamiento en línea.

La incorporación de un grupo control se basa principalmente en el argumento de que, para encontrar evidencias robustas acerca del efecto del FC en la adquisición de las estructuras tratadas y en el mejoramiento de la precisión gramatical, es necesaria la comparación con estudiantes que no reciben feedback. Los estudios que fracasan en este aspecto no entregan evidencia de la efectividad del FC (Bitchner, Young y Cameron, 2005; Bitchener, 2008; Ferris, 1999, 2004; Truscott, 1996, 2004). El diseño cautela que cada una de las instancias de evaluación, así como también las tareas escritas a realizar y el tiempo de trabajo sean idénticos para los grupos experimentales y control.

\section{FIGURA 1}

Modelo de investigación en FCE en el proceso de escritura de una L2

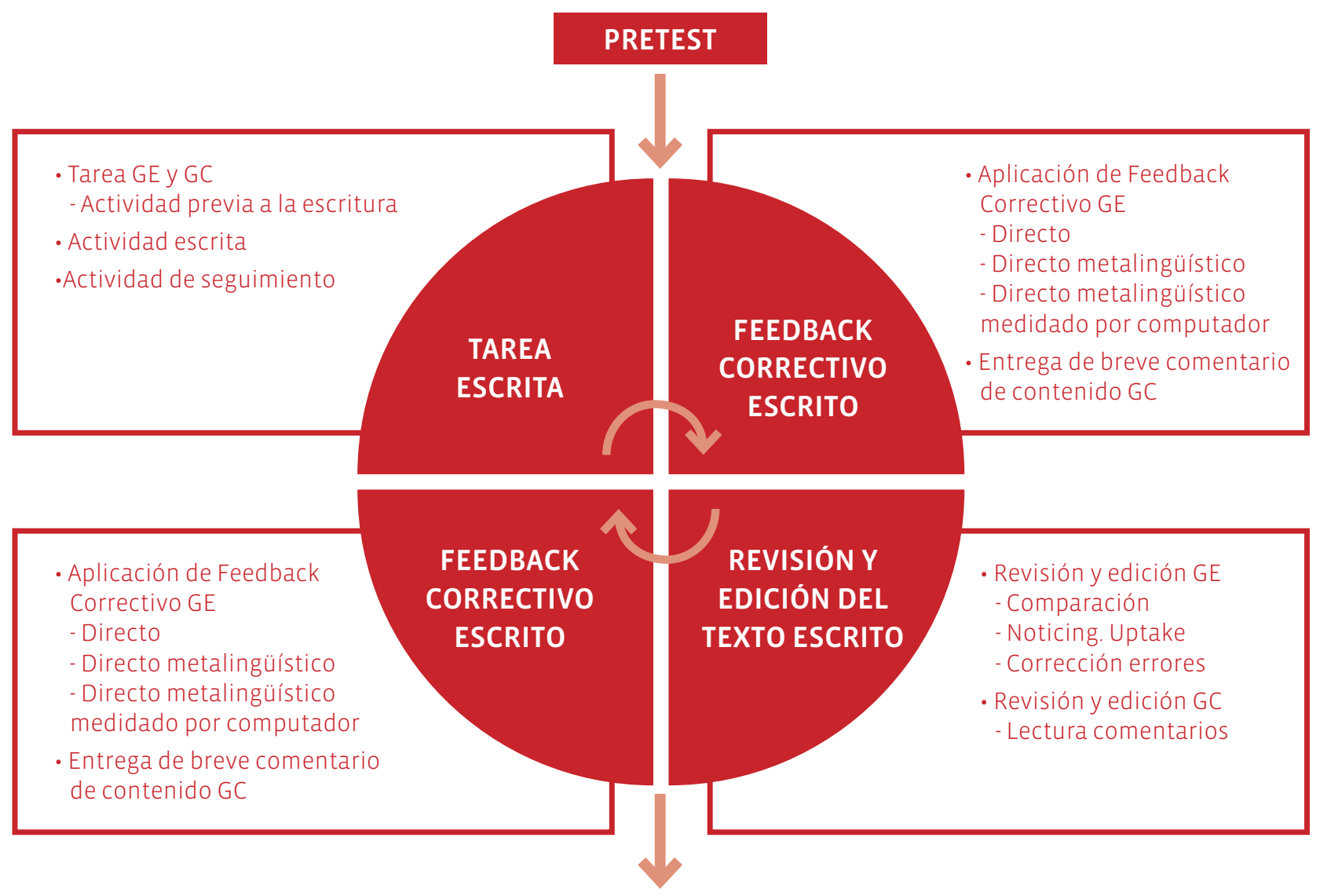

\section{POSTEST 1}

POSTEST 2 
Una de las fortalezas de este diseño es la forma en que se mide la adquisición de las estructuras. Se incorpora una medida longitudinal de mejoramiento de la precisión gramatical por medio de un pretest, postest inmediato y postest diferido (Bitchener, 2008). El postest inmediato se administra para minimizar los posibles efectos de otras variables intervinientes una vez realizado el tratamiento. El postest inmediato es una medición válida en el estudio, pero se requiere de un pretest con el cual se puedan comparar los resultados. El postest diferido es usado para medir la retención de las estructuras en el tiempo, y aunque no es posible eliminar los efectos de las variables intervinientes entre el postest inmediato y el diferido, de todas formas su utilización es una medida válida del nivel de retención observado en el postest inmediato. Cada una de estas instancias de evaluación considera un test de corrección de errores y la escritura de un texto nuevo de libre expresión; los textos deben ser de las mismas características textuales, de dificultad y de extensión léxica para cautelar que las comparaciones textuales sean válidas.

Una característica importante reflejada en el modelo es la direccionalidad del feedback correctivo escrito; se opta por el FC directo en contraposición al FC indirecto. La distinción entre FC directo e indirecto ha sido un tanto problemática. Ellis y otros (2008) señalan que desde una perspectiva práctica es poco probable que los profesores estén bastante familiarizados con la interlengua de sus estudiantes para tomar decisiones acerca de si es mejor corregir en forma directa o indirecta los errores de escritura. En consecuencia, desde una perspectiva pragmática, esta distinción no es la que necesita ser investigada. Se opta por el FC directo porque es el que tiene mayor relevancia para la adquisición de segundas lenguas. Se replica lo estudiado por otros investigadores (Bitchener, Young y Cameron, 2005; Sheen, 2007; Bitchener, 2008) en torno a la aplicación de feedback correctivo directo y directo metalingüístico, y se incorpora el feed- back directo mediado por computador. El feedback directo supone la entrega de la estructura correcta; en cambio, el metalingüístico incorpora alguna clave acerca de la naturaleza del error que ha sido cometido, así como la corrección necesaria; por otra parte, el feedback mediado por computador realiza la corrección en referencia a la interacción y a la negociación. Estas dos últimas estrategias de FC apelan al conocimiento explícito ayudando a los estudiantes a entender la naturaleza del error que se ha cometido (Ellis y otros, 2008; Sheen, 2007, 2011).

En este modelo, entonces, los tres tipos de feedback correctivo escrito que se proponen se definen de la siguiente manera:

1. Directo solamente: Esta opción de corrección le entrega al estudiante la forma correcta; se tarja la forma errónea y se inserta la forma correcta.

2. Directo metalingüístico: Se refiere a la entrega de la forma correcta con un acompañamiento de una explicación al pie de página en forma de regla gramatical.

3. Mediado por computador: Se utiliza alguna forma de comunicación escrita entre profesor y estudiante, utilizando el computador como mediación entre ambos, la que se centra en los errores de las estructuras.

Como se ha mencionado anteriormente existe también un comentario, que se le entrega al grupo control. Este consiste en información general acerca del contenido del texto. Esto obedece a una cuestión ética (Ferris, 2010; Sheen, 2011); no es posible en un contexto de aprendizaje no entregar algún tipo de retroalimentación acerca de una tarea realizada; lo que sí se debe cautelar es que esta información no esté dirigida al foco del tratamiento de feedback correctivo. Los comentarios pueden ser de la siguiente naturaleza:

- The message has been communicated fairly well.

- The text is clear and mostly convincing. 
Otro tema importante a destacar en este modelo es la integración de diferentes puntos de vista de investigación en torno al FC escrito. Se acoge lo propuesto por la investigación en ASL, en relación a si el FC ayuda a los estudiantes a adquirir las estructuras objeto de estudio en el tiempo; lo propuesto por los investigadores de CMC, quienes se preguntan si los ambientes tecnológicos constituyen un contexto similar o superior al presencial para el aprendizaje y adquisición de una segunda lengua, y, finalmente, lo propuesto por la investigación en producción escrita, que se pregunta si el FC escrito ayuda a los estudiantes a desarrollarse como escritores más eficientes (Ellis y otros, 2008; Ferris, 2010; Sheen, 2011).

El modelo funciona de la siguiente manera. Una vez tomado el pretest se inicia un ciclo de trabajo que comienza con una tarea que incorpora una actividad de preparación (prewriting) para la actividad escrita propiamente tal que será objeto de FC y una actividad de seguimiento (follow-up). A continuación se inicia el tratamiento de FC escrito en las modalidades descritas anteriormente para los grupos experimentales y el no feedback, sólo un comentario general, para el grupo control. Una vez entregado el FC se trabaja sobre el mismo texto, revisando las correcciones de acuerdo a la modalidad de FC recibida y al comentario general que recibe el grupo control; se edita el mismo texto y se reescribe para ser sometido a FC nuevamente para los grupos experimentales y a un comentario general para el grupo control. En la siguiente etapa se repite la entrega de FC escrito para las diferentes condiciones experimentales y el comentario general sobre el texto corregido para el grupo control. Posteriormente se inicia un nuevo ciclo de trabajo con una nueva actividad escrita que tiene como tarea central la escritura de un texto nuevo. El número de ciclos de aplicación del modelo va a depender del contexto y de las características lingüísticas de los estudiantes; sin embargo, se recomienda, especialmente en contextos escolares, entre dos a tres ciclos de trabajo. Esto se fundamenta en que este proceso puede tener efectos beneficiosos para los estudiantes ya que tienen mayor tiempo para pensar, desarrollar sus tareas, darse cuenta de los errores que cometen, procesar las correcciones y generar reparaciones y modificaciones de sus textos; pero también puede decaer su efectividad si la aplicación es demasiado prolongada.

En cuanto al tipo de actividad escrita y su dificultad, esto va a depender del nivel de competencia comunicativa que tengan los estudiantes de la muestra. Sin embargo, las tareas concebidas a priori son aquellas que promueven la escritura libre a partir de un estímulo concreto, una pieza imaginativa, coherente y significativa para el estudiante dentro de su contexto cotidiano de aprendizaje. Se trata justamente de evitar actividades aisladas que estén lejanas a su quehacer habitual. Un ejemplo de aquello podría ser una carta dirigida a algún personaje de un libro leído en la clase de lenguas en el que se demuestre algún sentido de tipo de texto. Por esto mismo, se incorpora una actividad de preparación (prewriting) que consiste en un ejercicio que genere las condiciones temáticas y léxicas que le permitan al estudiante situar el contexto en el cual va a desarrollar su texto escrito; es una forma de iniciar gradual y metódicamente la tarea como un todo; esta actividad puede consistir en la completación de cuadros o tablas como reacción a un estímulo visual o escrito, así como también una discusión entre pares para consolidar vocabulario y experiencias afines. De esta misma forma, la actividad de seguimiento (follow-up) es una actividad complementaria, de cierre, que le permite al estudiante gradualmente finalizar la tarea; se puede realizar para tal efecto algún juego léxico como un puzle o un ejercicio tipo cloze.

\section{Comentarios finales y proyecciones del modelo de investigación en FC escrito}

A pesar de la controversia que ha existido en torno al rol que tiene el FC escrito en la escritura 
de una L2 (Truscott, 1996; Ferris, 1999), de las diferencias metodológicas de los estudios de ASL y de producción escrita, de la aparición emergente de nuevas líneas de investigación como el uso de la tecnología en el FC, es importante señalar que un número cada vez mayor de investigaciones han mejorado el nivel de diseño, han obtenido resultados positivos y por lo tanto se han convertido en un serio reto para lo expuesto por Truscott (1996, 1999, 2001, 2004, 2007, 2009) en relación a que el FC no es gravitante en la adquisición de una L2 y que además es dañino para el desarrollo de la habilidad escrita en una segunda lengua.

Aquellos interesados principalmente en el FC desde la perspectiva de la producción escrita en una L2 deben preguntarse si la evidencia concluyente obtenida en los últimos años por los estudios rigurosamente diseñados puede ser utilizada para apoyar la continuación de las prácticas actuales y si tal evidencia ayuda a mejorar lo que los investigadores de escritura realizan actualmente (Ferris, 2010). Del mismo modo, los investigadores de ASL se deben cuestionar cómo sus diseños y hallazgos reflejan la realidad educacional que rodea la compleja tarea de escribir en una $L 2$, en una diversidad de contextos, con sujetos con intereses diversos. Al mismo tiempo, los investigadores de FC electrónico deben intentar descubrir cómo el feedback mediado por computador puede ser utilizado como parte del sistema completo de feedback correctivo, que incorpora modalidades presenciales y mediadas por tecnología, así como la participación de pares, expertos e investigadores. Estos tres enfoques tienen un gran potencial para avanzar en las prácticas investigativas y en la generación de conocimiento. Esto no se debe tomar como una competencia de aproximaciones a la temática de la corrección de los errores, sino como una complementación de fortalezas para encontrar evidencia más contundente acerca de los beneficios del FC.

Tomando muy en cuenta lo anteriormente mencionado en relación a la complementación que puede haber entre las líneas de investigación en FCE antes mencionadas, en este artículo se ha propuesto un modelo teórico de investigación de FCE que combina la metodología rigurosa y consistente que se ha venido desarrollando en los últimos años en ASL, la pertinencia de la aplicación de los resultados a situaciones educacionales reales y la incorporación del feedback mediado por computador como parte integral del sistema completo de feedback.

En el modelo, los estudiantes producen un texto (pretest), inician un ciclo iterativo que comienza con una tarea escrita, reciben un tipo de tratamiento de feedback correctivo, uno de ellos electrónico, y luego producen textos nuevos (postest y postest diferido), todo lo anterior dentro de un proceso de escritura que promueve la revisión y edición de textos. El modelo presupone, entre otras cosas, que el tiempo que los estudiantes tienen para reflexionar sobre el feedback y procesar la corrección escrita al revisar y editar sus textos genera uptake ${ }^{4}$ y reparaciones de las estructuras objeto de feedback, y de esta forma se promueve el desarrollo de habilidades escritas, así como también adquisición de la segunda lengua.

Para terminar, vale destacar que el objetivo final de la investigación en lingüística aplicada a la enseñanza y aprendizaje de segundas lenguas es la generación de conocimiento que les entregue a los profesores de lengua, a los estudiantes y a los investigadores la posibilidad de entender las formas de aprendizaje y de adquisición de una L2 y de esta manera contribuir al mejoramiento de los procesos involucrados en aquello. Sin embargo, no es suficiente focalizarse en las

4 Uptake ha sido usualmente definido como la respuesta inmediata que el hablante da a partir del feedback recibido, lo que ocurre en uno o dos turnos después del feedback (Loewen, 2007). 
necesidades de los estudiantes y los procesos de aprendizaje, porque también es necesario centrarse en el desarrollo de conocimiento acerca de cómo entregar feedback efectivo (Hyland, 2010). El modelo aquí propuesto busca indagar en cómo la aplicación del FC contribuye a la integración y colaboración de líneas de investigación en función de estos propósitos y nos ayuda a expandir el conocimiento y comprensión de cómo distintas formas de feedback son capaces de contribuir al desarrollo de los estudiantes de segundas lenguas como escritores exitosos.

\section{Bibliografía citada}

Barrientos, Fernanda, Anita Ferreira y Pedro SalCEDO, 2012: "Modelado del estudiante para el STI ELE-TUTOR: diseño de un componente adaptativo para apoyar la competencia lingüística del español como lengua extranjera”, Boletín de Filología 47 (1), 11-32.

Bitchener, John, Stuart Young y Denise Cameron, 2005: "The effect of different types of corrective feedback on ESL student writing", Journal of Second Language Writing 9, 227-258.

Bitchener, John, 2008: "Evidence in support of corrective feedback", Journal of Second Language Writing 17, 102-118.

Bitchener, John y Ute $\mathrm{K}_{\mathrm{NOCH}}$, 2008: "The value of written corrective feedback for migrant and international students", Language Teaching Research 12, 409-431.

Chomsky, Noah, 1986: Knowledge of language: its nature, origin and use, New York: Praeger.

Cook, Vivian, 1988: Chomsky's universal grammar. An introduction, Oxford: Basil Blackwell.

DeKeyser, Robert, 1998: "Beyond focus on form: Cognitive perspectives on learning and practicing second language grammar" en Catherine Doughty y Jessica Williams (eds.): Focus on form in classroom second language acquisition, Cambridge: Cambridge University Press, 42-63.
DeKeyser, Robert, 2003: "Implicit and explicit learning" en Catherine Doughty y Michael Long (eds.): Handbook of second language acquisition, Oxford: Blackwell, 313-348.

DiGıovanni, Elaine y Girija Nagaswami, 2001: "Online peer review: An alternative to face-to-face?”, ELT Journal 5 (3), 263-272.

Doughty, Catherine y Elizabeth Varela, 1998: "Communicative focus on form" en Catherine Doughty y Jessica Williams (eds.): Focus on form in classroom second language acquisition, Cambridge: Cambridge University Press, 114-138.

Doughty, Catherine y Jessica Williams, 1998: "Pedagogical choices in focus on form" en Catherine Doughty y Jessica Williams (eds.): Focus on form in classroom second language acquisition, Cambridge: Cambridge University Press, 197-261.

EluIs, Rod, 1997: SLA Research and Language Teaching, Oxford: Oxford University Press.

EluIs, Rod, 2005: "Principles of instructed language learning”, System 33 (2), 209-224.

ElLIs, Rod, 2009: "A Typology of written corrective feedback types”, ELT Journal 63 (2), 97-107.

EluIs, Rod, 2010: "A framework for investigating oral and written corrective feedback", Studies in Second Language Acquisition 32 (2), 335-349.

EluIs, Rod y otros, 2008: "The Effects of Focused and Unfocused Written Corrective Feedback in an English as a Foreign Language Context", System 36, 353-371.

FerreIRA, Anita, 2003: Feedback strategies for second language teaching with implications for Intelligent Tutorial Systems. Ph.D. Thesis, University of Edinburgh, Edinburgh, UK. Free Ling 2.2. FerreIra, Anita, 2006: "Estrategias de feedback positivo y correctivo en el español como lengua extranjera”, Revista Signos. Estudios de Lingüística, vol. 39, n. 62, 379-406.

Ferreira, Anita, 2007: "Estrategias efectivas de feedback correctivo para el aprendizaje de lenguas asistido por computadores", Revista Signos. Estudios de Lingüística 40 (65), 521-544. 
Ferreira, Anita, Johanna Moore y Chris Mellish, 2007: "A study of feedback strategies in foreign language classrooms and tutorials with implications for Intelligent Computer Assisted language learning systems". International Journal of Artificial Intelligence in Education 17 (4), 389-422.

Ferreira, Anita y John Atkinson, 2009: "Designing a feedback component of an intelligent tutoring system for foreign language, Knowledge-Based Systems Journal", Elsevier 22 (7), 496-501.

Ferreira, Anita y Gabriela Kotz, 2010: "Ele-TUtOR Inteligente: Un analizador computacional para el tratamiento de errores gramaticales en Español como Lengua Extranjera”, Revista Signos. Estudios de Lingüística 43 (73), 211-236.

Ferrelra, Anita y otros, 2012: "La Arquitectura de ELE-TUTOR: Un sistema tutorial inteligente para el Español como Lengua Extranjera”, Revista Signos. Estudios de Lingüística 45 (79), 102-131.

FerRIS, Dana, 1999: "The case for grammar correction in L2 writing classes, a response to Truscott”, Journal of Second Language Writing 8, 1-12. FERRIS, Dana, 2002: Treatment of error in second language student writing, Michigan: The University of Michigan Press.

FERRIS, Dana, 2003: Response to student writing: Implications for second language students, Mahwah, NJ: Erlbaum.

FerRIS, Dana, 2004: “The 'grammar correction' debate in L2 writing: Where are we, and where do we go from here? (and what do we do in the meantime?)", Journal of Second Language Writing $13,49-62$.

FerRIS, Dana, 2006: "Does error feedback help student writers? New evidence on the short- and long-term effects of written error correction" en Ken Hyland y Fiona Hyland (eds.): Feedback in second language writing: Contexts and issues, Cambridge: Cambridge University Press, 81-104. FerRIs, Dana, 2010: "Second language writing research and written corrective feedback in SLA Intersections and Practical Applications", Studies in Second Language Acquisition 32,181-201.
HyLAND, Fiona, 2003: "Focusing on form: student engagement with teacher feedback", System, vol. 31, n. 2, 217-230

Hyland, Fiona, 2010: "Future directions in feedback on second language writing: Overview and Research Agenda", International Journal of English Studies, vol. 10, n. 2, 171-182.

Hyland, Ken y Fiona Hyland, 2006: "Feedback on second language students' writing”, Language Teaching 39, 83-101.

Kotz, Gabriela y Anita FerReIRA, 2013: "La precisión gramatical mediada por la tecnología: El análisis y tratamiento automático de errores", Revista Literatura y Lingüística 27, 217-240.

Krashen, Stephen, 1981: Second language acquisition and second language learning, Oxford: Pergamon.

Krashen, Stephen, 1982: Principles and practices in second language acquisition, Oxford: Pergamon.

Krashen, Stephen, 1985. The input hypothesis: Issues and implications, London: Longman.

LoEwen, Shawn, 2007: "The prior and subsequent use of forms targeted in incidental focus on form" en Sandra Fotos y Hossein Nassall (eds.): Form focused instruction and teacher education: Studies in honor of Rod Ellis, Oxford: Oxford University Press, 101-116.

Loewen, Shawn y Rosemary ERLam, 2006: "Corrective feedback in the chatroom: An experimental study", Computer Assisted Language Learning 19 (1), 1-14.

Long, Michael, 1991: "Focus on form: A design feature in language teaching methodology" en Kees de Bot, Ralph Ginsberg y Claire Kramsch (eds.): Foreign language research in cross-culturalperspectives, Amsterdam/Philadelphia: John Benjamins Publishing Company, 39-52.

LoNG, Michael, 1996: "The role of linguistic environment in second language acquisition" en William Ritchie y Tej Bahtı (eds.): Handbook of se- 
cond language acquisition, New York: Academic Press, 413-468.

Mıton, John, 2006: "Resource-Rich Web-Based Feedback: helping learners become independent writers" en Ken Hyland y Fiona Hyland (eds.): Feedback in second language writing: Contexts and issues, Cambridge: Cambridge University Press, 123-139.

Pellettierl, Jill, 2000: "Negotiation in cyberspace: The role of chatting in the development of grammatical competence" en Mark Warschauer y Richard Kern (eds.): Network-Based LanguageTeaching: Concepts and Practice, Cambridge: Cambridge University Press, 59-86.

Pienemann, Manfred, 1989: "Is language teachable?”, Applied Linguistics 10 (1), 52-79.

Pienemann, Manfred, 1998: Language Processing and Second Language Development: Processability theory, Amsterdam/Philadelphia: John Benjamins Publishing Company.

Sawyer, Mark y Leyla Ranta, 2001: "Aptitude, individual differences, and instructional design" en Peter Robinson (ed.): Cognition and second language instruction, Cambridge: Cambridge University Press, 319-353.

Schmidt, Richard, 1990: "The role of consciousness in second language learning", Applied Linguistics 11 (2), 129-158.

SchmidT, Richard, 1994: "Deconstructing consciousness in search of useful definitions for applied Linguistics", AlLA Review 11, 11-26.

SchmidT, Richard, 2001: "Attention" en Peter RobINson (ed.): Cognition and second language instruction, Cambridge: Cambridge University Press, 3-32.

Schwartz, Benjamin, 1986: "The epistemological status of second language acquisition", Second Language Research 2, 120-159.

Sheen, YoungHee, 2007: "The effect of focused written corrective feedback and language apti- tude on ESL learners' acquisition of articles", TESOL Quarterly 41, 255-283.

SHeEN, YoungHee, 2011: Corrective Feedback Individual Differences and Second Language Learning, New York: Springster.

SwaIn, Merril, 1985: "Communicative competence: Some roles of comprehensible input and comprehensible output in its development" en Susan Gass y Caroline Madden (eds.): Input in second language acquisition, Rowley, MA: Newbury House, 235-253.

TRuscotT, John, 1996: "The case against grammar correction in L2 writing classes", Language Learning 46 (2), 327-369.

Truscott, John, 1999: "The case for 'The case against grammar correction in L2 writing classes': A Response to Ferris", Journal of Second Language Writing 8 (2), 111-122.

TRuscotT, John, 2004: "Evidence and conjecture on the effects of correction: a response to Chandler", Journal of Second Language Writing 13, 337343.

TRuscotT, John, 2007: "The effect of error correction on learners' ability to write accurately", Journal of Second Language Writing 16 (4), 255-272.

TRuscotT, John, 2009: "Arguments and appearances: a response to Chandler", Journal of Second Language Writing 18, 59-60.

Van Beuningen, Catherine, 2010: "Corrective Feedback in L2 Writing: Theoretical Perspectives, Empirical Insights, and Future Directions", International Journal of English Studies 10 (2), 1-27.

Ware, Paige y Mark Warschauer, 2006: "Electronic feedback and second language writing", Ken Hyland y Fiona Hyland (eds.): Feedback in second language writing: Contexts and issues, Cambridge: Cambridge University Press, 105-122. 